\title{
Application of slice regularity to functions of a dual-quaternionic variable
}

\begin{abstract}
Kim Ji Eun
In this paper, we present the algebraic properties of dual quaternions and define a slice regularity of a dual quaternionic function. Since the product of dual quaternions is non-commutative, slice regularity is derived in two ways. Thereafter, we propose the Cauchy-Riemann equations and a power series corresponding to dual quaternions.

Key words and phrases: dual number, quaternion, Cauchy-Riemann equation, slice regular function, Clifford analysis.
\end{abstract}

Dongguk University, Gyeongju 38066, South Korea

E-mail: jeunkimepusan.ac.kr

\section{Introduction}

Many fields of analysis have explored the theory of quaternionic valued functions of quaternion variables, which consider holomorphic functions of complex variables. R. Fueter [4] first defined the differential operator as follows

$$
\frac{\partial}{\partial \bar{q}}=\frac{1}{4}\left(\frac{\partial}{\partial x_{0}}+i \frac{\partial}{\partial x_{1}}+j \frac{\partial}{\partial x_{2}}+k \frac{\partial}{\partial x_{3}}\right)
$$

called the Cauchy-Fueter operator and constructed a space consisting of solutions of the equation expressed by the Cauchy-Fueter operator. Since then, based on [3], many results on properties and applications of solutions of the Cauchy-Fueter equation have been reported (see $[1,11,12])$. However, a function defined as a solution of the Cauchy-Fueter equation expresses the identity as the equation $f(q)=q$, but there exists the situation that polynomials and series of this function are not regular in quaternions. To solve these problems, consider the definition of the Cullen regular function that was proposed and developed by C.G. Cullen [2]. According to this definition, polynomials and power series of the form $\sum_{n=0}^{\infty} q^{n} a_{n}$, where $q \in \mathbb{H}$, being the set of quaternions, and $a_{n}$ are constants in $\mathbb{H}$, are regular. In addition, R. Fueter defined the class of holomorphic functions over $\mathbb{H}$ and expressed polynomials and power series of quaternionic variables, based on that class. Thereafter, G. Laville and I. Ramadanoff [9] developed the theory of holomorphic functions over $\mathbb{H}$ and constructed the definition of holomorphic Cliffordian functions. Since the Laplacian equation denoted by the differential operator $\frac{\partial}{\partial \bar{q}} \Delta$ has solutions that are holomorphic functions over $\mathbb{H}$, the set of Cullen regular functions and the set of Fueter regular functions are not equivalent. The class of Cullen regular functions consists of all solutions of a generalized Cauchy-Riemann system of equations, which proposes polynomials and series expansion in $\mathbb{H}$. To provide the generalized Cullen definition, 
let $\mathrm{S}$ be the unit sphere of a pure imaginary quaternion, i.e.

$$
\mathrm{S}=\left\{q=i x_{1}+j x_{2}+k x_{3} \mid x_{1}^{2}+x_{2}^{2}+x_{3}^{2}=1\right\} .
$$

If $I \in \mathbb{H}$, then $I^{2}=-1$ and the elements of $S$ are called imaginary units. G. Gentili and D.C. Struppa $[5,6]$ developed properties and theories of slice regularity by using the generalized Cullen definition. Building upon $[5,6]$, this study extends the notions and properties of slice regularity in dual quaternions. J.E. Kim et al. $[7,8]$ investigated the extended the regularity of dual quaternionic functions and represented dual quaternionic functions by using various forms such as their polar form in Clifford analysis. This study shows that the generalized Cullen definition can be applied to the set of dual quaternions and that theories of slice regular functions can be applied to dual quaternions. Therefore, in this study, we provide the algebraic properties of dual quaternions and define a slice regularity of a dual quaternionic function. Due to the non-commutativity of the product of dual quaternions, slice regularity induces the corresponding Cauchy-Riemann equations and power series in dual quaternions.

\section{Preliminaries}

In this section, we provide the basic definitions and algebraic properties of dual quaternions. We recall that the set of dual quaternions $\mathbb{D}_{q}$ is denoted by

$$
\mathbb{D}_{q}=\left\{p \mid p=x_{0}+i x_{1}+j x_{2}+k x_{3}, \quad x_{t} \in \mathbb{R}, \quad t=0,1,2,3\right\},
$$

where the imaginary unit $i$ and dual unit $j$ with the condition that $i j=k$ satisfy the following properties:

$$
i^{2}=-1, \quad j^{2}=0, \quad i j=k=-j i, \quad j k=k j=0, \quad k i=j=-i k .
$$

Given the existence of these units, if we consider an example by means of the matrix form such as

$$
1=\left(\begin{array}{ll}
1 & 0 \\
0 & 1
\end{array}\right), \quad i=\left(\begin{array}{cc}
i & 0 \\
0 & -i
\end{array}\right), j=\left(\begin{array}{ll}
0 & 1 \\
0 & 0
\end{array}\right) \quad \text { and } \quad k=\left(\begin{array}{cc}
0 & i \\
0 & 0
\end{array}\right),
$$

then these matrices satisfy equations (1).

From the properties of units for dual-quaternions, we define the conjugate of a dual-quaternion $p$ as $p^{*}=x_{0}-i x_{1}-j x_{2}-k x_{3}$. Then, its modulus $|\cdot| \mathbb{D}$ is given by

$$
|p|_{\mathbb{D}}^{2}=p p^{*}=x_{0}^{2}+x_{1}^{2}
$$

and the real and imaginary part of $p$ are denoted by $\operatorname{Re}(p)=x_{0}$ and $\operatorname{Im}(p)=i x_{1}+j x_{2}+k x_{3}$, respectively. If the coefficients $x_{0}$ of 1 and $x_{1}$ of $i$ are both nonzero, we have the multiplicative inverse of $p$, denoted by

$$
p^{-1}=\frac{p^{*}}{|p|_{\mathbb{D}}^{2}}
$$

Let $\mathbb{L}$ be a set of pure imaginary dual-quaternions, denoted by

$$
\mathbb{L}=\left\{p \in \mathbb{D}_{q}|\operatorname{Re}(p)=0,| \operatorname{Im}(p) \mid=1\right\},
$$

where $|\operatorname{Im}(p)|=\left|x_{1}\right|$, i.e. $|\operatorname{Im}(p)|$ is isomorphic to a projection $\pi_{i}: \mathbb{D}_{q} \rightarrow \mathbb{R}_{i}$, where $\mathbb{R}_{i}$ is the set of real numbers that are coefficients of the unit $i$. Any $I \in \mathbb{L}$ satisfies the equation $I^{2}=-1$. Consider the complex plane

$$
\Lambda_{I}=\{x+I y \mid x, y \in \mathbb{R}\}
$$


For any $p \in \Lambda_{I}$, there exist unique values of $x, y \in \mathbb{R}$ such that $p=x+I y$. Since the elements of $\Lambda_{I}$ satisfy the condition that the square of each element is -1 , we obtain the differential operators

$$
D_{I}^{*}:=\frac{1}{2}\left(\frac{\partial}{\partial x}+I \frac{\partial}{\partial y}\right) \quad \text { and } \quad D_{I}=\frac{1}{2}\left(\frac{\partial}{\partial x}-I \frac{\partial}{\partial y}\right) .
$$

Using these operators, we define other functions that are described in the following section.

\section{Slice regular function in dual-quaternions}

To define the slice regular function in $\mathbb{D}_{q}$, we introduce the following definition.

Definition 1 ([10]). A subset $\Omega$ of $\mathbb{D}_{q}$ is said to be a dual subset if there exists a subset $\mathcal{O} \subset \mathbb{R}^{2}$ such that $\Omega=\mathcal{O} \times \mathbb{R}^{2}$. The subset $\mathcal{O}$ is called the generator of $\Omega$. The subset $\Omega$ is said to be an open dual subset of $\mathbb{D}_{q}$ if the generator of $\Omega$ is an open subset of $\mathbb{R}^{2}$. The set $\Omega$ is said to be a closed dual subset of $\mathbb{D}_{q}$ if its complementary is an open subset of $\mathbb{D}_{q}$. The set $\Omega$ is said to be a connected dual subset of $\mathbb{D}_{q}$ if its generator is a connected subset of $\mathbb{R}^{2}$.

Let $f: \Omega \rightarrow \mathbb{D}_{q}$ be a function with the units used in $\mathbb{D}_{q}$ such that

$$
f(p)=u_{0}+i u_{1}+j u_{2}+k u_{3}
$$

where $u_{r}=u_{r}\left(x_{0}, x_{1}, x_{2}, x_{3}\right), r=0,1,2,3$, are real-valued functions. The function $f$ is called a dual quaternionic function.

Next, we provide the definition of a slice regular function in dual-quaternions.

Definition 2. Let $\Omega$ be a domain in $\mathbb{D}_{q}$. A function $f: \Omega \rightarrow \mathbb{D}_{q}$ is said to be slice left regular (SLR), if its restriction $f_{I}: \Omega \cap \Lambda_{I} \rightarrow \mathbb{D}_{q}$ has a continuous partial derivative and satisfies the equation $D_{I}^{*} f(p)=D_{I}^{*} f(x+I y)=0$ for $p=x+I y \in \Omega \cap \Lambda_{I}$, that is

$$
D_{I}^{*} f(p)=\frac{1}{2}\left(\frac{\partial f_{I}(p)}{\partial x}+I \frac{\partial f_{I}(p)}{\partial y}\right)
$$

on $\Omega \cap \Lambda_{I}$. In addition, $f$ is said to be slice right regular (SRR), if $f_{I}$ is differentiable and satisfies $f(p) D_{I}^{*}=f(x+I y) D_{I}^{*}=0$, that is we have

$$
f(p) D_{I}^{*}=\frac{1}{2}\left(\frac{\partial f_{I}(x+I y)}{\partial x}+\frac{\partial f_{I}(x+I y)}{\partial y} I\right)
$$

on $\Omega \cap \Lambda_{I}$.

Example. We consider the power series of $p=x+I y \in \Omega \cap \Lambda_{I}$, that is, for $p=x+I y \in \Omega \cap \Lambda_{I}$

$$
f(p)=p^{n}=\sum_{k=0}^{n}\left(\begin{array}{c}
n \\
2 k
\end{array}\right)(-1)^{k} x^{n-2 k} y^{2 k}+I \sum_{k=0}^{n}\left(\begin{array}{c}
n \\
2 k+1
\end{array}\right)(-1)^{k} x^{n-2 k-1} y^{2 k+1} \text {. }
$$

Since $f$ satisfies

$$
\begin{aligned}
D_{I}^{*} f(p)= & \sum_{k=0}^{n}\left(\begin{array}{c}
n \\
2 k
\end{array}\right)(-1)^{k} x^{n-2 k} y^{2 k}+I \sum_{k=0}^{n}\left(\begin{array}{c}
n \\
2 k+1
\end{array}\right)(-1)^{k} x^{n-2 k-1} y^{2 k+1} \\
= & \sum_{k=0}^{n}\left(\begin{array}{c}
n \\
2 k
\end{array}\right)(-1)^{k}(n-2 k) x^{n-2 k-1} y^{2 k}+I \sum_{k=0}^{n}\left(\begin{array}{c}
n \\
2 k+1
\end{array}\right)(-1)^{k}(n-2 k-1) x^{n-2 k-2} y^{2 k+1} \\
& +I\left\{\sum_{k=1}^{n}\left(\begin{array}{c}
n \\
2 k
\end{array}\right)(-1)^{k} 2 k x^{n-2 k} y^{2 k-1}+I \sum_{k=0}^{n}\left(\begin{array}{c}
n \\
2 k+1
\end{array}\right)(-1)^{k}(2 k+1) x^{n-2 k-1} y^{2 k}\right\},
\end{aligned}
$$


where

$$
\left(\begin{array}{c}
n \\
2 k
\end{array}\right)(n-2 k)=\left(\begin{array}{c}
n \\
2 k+1
\end{array}\right)(2 k+1) \quad \text { and } \quad\left(\begin{array}{c}
n \\
2 k+1
\end{array}\right)(n-2 k-1)=\left(\begin{array}{c}
n \\
2(k+1)
\end{array}\right) 2(k+1)
$$

for $k=0,1,2, \ldots$ Thus, we obtain

$$
\begin{aligned}
D_{I}^{*} f(p)= & \left\{\sum_{k=0}^{n}\left(\begin{array}{c}
n \\
2 k
\end{array}\right)(-1)^{k}(n-2 k) x^{n-2 k-1} y^{2 k}-\sum_{k=0}^{n}\left(\begin{array}{c}
n \\
2 k+1
\end{array}\right)(-1)^{k}(2 k+1) x^{n-2 k-1} y^{2 k}\right\} \\
& +I\left\{\sum_{k=0}^{n}\left(\begin{array}{c}
n \\
2 k+1
\end{array}\right)(-1)^{k}(n-2 k-1) x^{n-2 k-2} y^{2 k+1}+\sum_{k=1}^{n}\left(\begin{array}{c}
n \\
2 k
\end{array}\right)(-1)^{k} 2 k x^{n-2 k} y^{2 k-1}\right\} \\
= & 0 .
\end{aligned}
$$

Therefore, the function $f$ can be called SLR.

Definition 3. Let $\Omega$ be a domain in $\mathbb{D}_{q}$ and let $f$ be a SLR function. The slice left derivative (SLD) $D_{I}$ of $f$ is defined as

$$
D_{I} f(x+I y)=\frac{1}{2}\left(\frac{\partial f_{I}(x+I y)}{\partial x}-I \frac{\partial f_{I}(x+I y)}{\partial y}\right)
$$

and the slice right derivative (SRD) $D_{I}$ of $f$ is defined as

$$
f(x+I y) D_{I}=\frac{1}{2}\left(\frac{\partial f_{I}(x+I y)}{\partial x}-\frac{\partial f_{I}(x+I y)}{\partial y} I\right) .
$$

Since the calculus processes of SLR and SRR are similar, for brevity, we mainly deal with the definition of SLR alone in this study.

Proposition 1. Let $\Omega$ be a domain in $\mathbb{D}_{q}$ and a function $f$ be SLR in $\mathbb{D}_{q}$. Then, the function $f$ satisfies the following equations

$$
D_{I} f(x+I y)=\frac{\partial f_{I}(x+I y)}{\partial x} \quad \text { or } \quad D_{I} f(x+I y)=-I \frac{\partial f_{I}(x+I y)}{\partial y} .
$$

Proof. Since $f$ is a SLR function, it satisfies

$$
D_{I}^{*} f(p)=\frac{1}{2}\left(\frac{\partial f_{I}}{\partial x}+I \frac{\partial f_{I}}{\partial y}\right)=0 .
$$

Therefore, we have

$$
D_{I} f(p)=\frac{1}{2}\left(\frac{\partial f_{I}}{\partial x}-I \frac{\partial f_{I}}{\partial y}\right)=\frac{\partial f_{I}}{\partial x} \quad \text { or } \quad D_{I} f(p)=-I \frac{\partial f_{I}}{\partial y}
$$

Thus, we obtain equation (2).

Proposition 2. Let $\Omega$ be a domain in $\mathbb{D}_{q}$. Let $f$ be a $S L R$ function that acts on $\Omega$. Then, the SLD of $f$, denoted by $D_{I}^{n} f: \Omega \rightarrow \mathbb{D}_{q}$, is SLR and it satisfies

$$
D_{I}^{n} f(x+I y)=\frac{\partial^{n} f(x+I y)}{\partial x^{n}}
$$

Proof. From Proposition 1, by means of induction, equation (3) is obtained. 
Proposition 3. Let $\Omega$ be a domain in $\mathbb{D}_{q}$. If $f$ is a SLR function, then the SLD of $f$ is also regular.

Proof. Since operators $D_{I}$ and $D_{I}^{*}$ commute with each other, they satisfy the equation $D_{I}^{*}\left(D_{I} f\right)=D_{I}\left(D_{I}^{*} f\right)$. In addition, since $f$ is SLR in $\Omega$, the function $f$ satisfies $D_{I}^{*} f(p)=0$. Thus, we obtain $D_{I}^{*}\left(D_{I} f\right)=D_{I}\left(D_{I}^{*} f\right)=0$.

Theorem 1. Let $\Omega$ be a domain in $\mathbb{D}_{q}$. If $f$ is a SLR function on $\Omega$, then for every $I, J \in \mathbb{L}$ there exist SLR functions $F, G: \Omega \cap \Lambda_{I} \rightarrow \Lambda_{I}$ such that for any $p \in \Lambda_{I}$ it satisfies

$$
f_{I}(p)=F(p)+G(p) J .
$$

Proof. Consider the restriction $f_{I}$ of $f$ to $\Omega \cap \Lambda_{I}$ such that $f_{I}(x+I y)=f_{0}+I f_{1}+J f_{2}+K f_{3}$, where $f_{t}: \mathbb{R}^{2} \rightarrow \mathbb{R}, t=0,1,2,3$, are real-valued functions and let $K:=I J$. Since $f$ is a SLR function, it satisfies $D_{I}^{*} f=0$. Hence, we have

$$
\begin{aligned}
0 & =\frac{1}{2}\left(\frac{\partial}{\partial x}+I \frac{\partial}{\partial y}\right)\left\{f_{0}(x, y)+I f_{1}(x, y)+J f_{2}(x, y)+K f_{3}(x, y)\right\} \\
& =\frac{1}{2}\left\{\frac{\partial f_{0}}{\partial x}+I \frac{\partial f_{1}}{\partial x}+J \frac{\partial f_{2}}{\partial x}+K \frac{\partial f_{3}}{\partial x}+I \frac{\partial f_{0}}{\partial y}-\frac{\partial f_{1}}{\partial y}+K \frac{\partial f_{2}}{\partial y}-J \frac{\partial f_{3}}{\partial y}\right\} \\
& =\frac{1}{2}\left\{\left(\frac{\partial f_{0}}{\partial x}-\frac{\partial f_{1}}{\partial y}\right)+I\left(\frac{\partial f_{1}}{\partial x}+\frac{\partial f_{0}}{\partial y}\right)+J\left(\frac{\partial f_{2}}{\partial x}-\frac{\partial f_{3}}{\partial y}\right)+K\left(\frac{\partial f_{3}}{\partial x}+\frac{\partial f_{2}}{\partial y}\right)\right\}
\end{aligned}
$$

This implies

$$
\left\{\begin{array} { l } 
{ \frac { \partial f _ { 0 } } { \partial x } = \frac { \partial f _ { 1 } } { \partial y } , } \\
{ \frac { \partial f _ { 1 } } { \partial x } = - \frac { \partial f _ { 0 } } { \partial y } , }
\end{array} \quad \text { and } \quad \left\{\begin{array}{l}
\frac{\partial f_{2}}{\partial x}=\frac{\partial f_{3}}{\partial y} \\
\frac{\partial f_{3}}{\partial x}=-\frac{\partial f_{2}}{\partial y}
\end{array}\right.\right.
$$

If we let $F=f_{0}+I f_{1}$ and $G=f_{2}+I f_{3}$, then $F$ and $G$ satisfy the equations $D_{I}^{*} F=0$ and $D_{I}^{*} G=0$. Thus, $F$ and $G$ are SLR functions, and we obtain $f_{I}=F+G J$.

The equations (4) that appeared in the above proof procedure are called corresponding Cauchy-Riemann equations, and they are derived from $D_{I}^{*} f_{I}=0$.

We now consider polynomials and power series in $\mathbb{D}_{q}$. The standard polynomial $p^{n} a_{n}$, with $a_{n}$ being dual-quaternions for each $n \in \mathbb{N}$, is regular. In addition, the sum of SLR functions is SLR. From these properties of regularity in $\mathbb{D}_{q}$, we obtain polynomials with dual-quaternionic coefficients on the right that are slice regular. We define the space of regular functions that have a uniform convergence on compact sets.

Theorem 2. Let $\Omega$ be a domain in $\mathbb{D}_{q}$. Let $f: \Omega \rightarrow \mathbb{D}_{q}$ be a SLR function on $\Omega$. Then, there exists

$$
f(p)=\sum_{n=0}^{\infty} p^{n} \frac{1}{n !} \frac{\partial^{n} f(0)}{\partial x^{n}}
$$

converging on $\Omega$.

Proof. Let $\mathfrak{D}_{I}$ be a disc centered at the origin with a radius $a>0$, where $a<R$. From Theorem 1 , we consider an integral representation for $f_{I}$ in $\mathfrak{D}_{I}$. Since $f_{I}=F+G J$, where both $F$ and $G$ are SLR in $\Omega \cap \Lambda_{I}$, for any $\zeta, p \in \Omega \cap \Lambda_{I}$, each of $F(p)$ and $G(p)$ is commutative with

$$
(\zeta-p)^{-1}=\frac{(\zeta-p)^{*}}{|\zeta-p|^{2}}
$$


where $\zeta=\tau_{0}+I \tau_{1}$ and $p=x_{0}+I x_{1}$ with $\tau_{t} \neq x_{t}, t=0,1$. Therefore, we write the following equations

$$
(\zeta-p)^{-1} F(p):=\frac{F(p)}{\zeta-p} \quad \text { and } \quad(\zeta-p)^{-1} G(p):=\frac{G(p)}{\zeta-p} .
$$

Hence, for any $p$ in $\mathfrak{D}_{I}$ we have

$$
f_{I}(p)=\frac{1}{2 \pi I}\left(\int_{b \mathfrak{D}_{I}} \frac{F(\zeta)+G(\zeta) J}{\zeta-p} d \zeta\right)=\frac{1}{2 \pi I}\left\{\int_{b \mathfrak{D}_{I}} \frac{F(\zeta)}{\zeta-p} d \zeta+\left(\int_{b \mathfrak{D}_{I}} \frac{G(\zeta)}{\zeta-p} d \zeta\right) J\right\} .
$$

Since $\zeta \in \Omega \cap \Lambda_{I}$, we write $\zeta^{-1}=\frac{1}{\zeta}$ if it is expressed with other elements of $\Lambda_{I}$.

$$
\begin{aligned}
f_{I}(p) & =\frac{1}{2 \pi I}\left\{\int_{b \mathfrak{D}_{I}} \frac{1}{1-\frac{p}{\zeta}} \frac{F(\zeta)}{\zeta} d \zeta+\left(\int_{b \mathfrak{D}_{I}} \frac{1}{1-\frac{p}{\zeta}} \frac{G(\zeta)}{\zeta} d \zeta\right) J\right\} \\
& =\frac{1}{2 \pi I}\left\{\sum_{n \in \mathbb{N}} p^{n} \int_{b \mathfrak{D}_{I}} \frac{F(\zeta)}{\zeta} d \zeta+\left(\sum_{n \in \mathbb{N}} p^{n} \int_{b \mathfrak{D}_{I}} \frac{G(\zeta)}{\zeta} d \zeta\right) J\right\} .
\end{aligned}
$$

Since the power series is regular in its domain of convergence, we have

$$
f_{I}(p)=\sum_{n \in \mathbb{N}} p^{n} \frac{1}{n !}\left(D_{I}^{n} F(0)+D_{I}^{n} G(0) J\right)=\sum_{n \in \mathbb{N}} p^{n} \frac{1}{n !} D_{I}^{n} f(0) .
$$

From Proposition 2, we have

$$
f_{I}(p)=\sum_{n \in \mathbb{N}} p^{n} \frac{1}{n !} D_{I}^{n} f(0)=\sum_{n \in \mathbb{N}} p^{n} \frac{\partial^{n} f(0)}{\partial x^{n}} .
$$

Since the function $f_{I}(p)$ can be expressed as a series for arbitrary $I \in \Lambda_{I}$, we obtain the condition that for any $I \in \Lambda_{I}$, the function $f$ has a series expansion such that equation (5) holds.

Example. Considering the definition of the exponential function in quaternion, we denote $\exp (p)=\exp (x)(\cos y+I \sin y)$. Since the exponential function of $p \in \Omega \cap \Lambda_{I}$ satisfies

$$
D_{I}^{*} \exp (p)=\exp (x)(\cos y+I \sin y)+I \exp (x)(-\sin y+I \cos y)=0
$$

for $p \in \Omega \cap \Lambda_{I}, \exp (p)$ is SLR. The exponential function $\exp (p)$ has $\frac{\partial^{n}}{\partial x^{n}} \exp (p)=\exp (p)$, and thus, $\exp (p)=\sum_{n=0}^{\infty} p^{n} \frac{1}{n !}$ can be obtained.

\section{Conclusion}

In this paper, the algebraic properties of dual quaternions is given and the slice regularity of a dual quaternionic function is defined. The interesting property of dual quaternions is that they can be expressed as the Galilean transformation in one quaternion equation. Since the multiplication of two dual quaternions is a dual quaternion, the set of dual quaternions form a division algebra under multiplication. Dual quaternions are useful mathematical tools for the rigid body motions in engineering and physics. The dual quaternions are applied as a number system to represent rigid transformations in three dimensions. Since the product of dual quaternions is non-commutative, when defining the notion of slice regularity, it is necessary to consider the order of the associated functional calculus. Also, before we consider power series expansions of slice regular functions of a dual quaternion variable, we need to introduce a new type of derivative for slice regular functions. Power series in the dual quaternion variable are more complicated than complex power series because of the non-commutativity of the quaternionic multiplication. In view of the non-commutativity and slice regularity, we propose the left(right)-Cauchy-Riemann equations and a power series corresponding to dual quaternions. 


\section{Acknowledgement}

This work was supported by the Dongguk University Research Fund of 2019.

\section{References}

[1] Adams W.W., Berenstein C.A., Loustaunau P., Sabadini I., Struppa D.C. Regular functions of several quaternionic variables and the Cauchy-Fueter complex. J. Geom. Anal. 1999, 9 (1), 1-15. doi:10.1007/BF02923085

[2] Cullen C.G. An integral theorem for analytic intrinsic functions on quaternions. Duke Math. J. 1965, 32 (1), 139 148. doi:10.1215/S0012-7094-65-03212-6

[3] Fueter R. Analytische funktionen einer quaternionenvariablen. Comment. Math. Helv. 1932, 4 (1), 9-20. (in German) doi:10.1007/BF01202702

[4] Fueter R. Die Funktionentheorie der Differentialgleichungen $\Delta u=0$ und $\Delta \Delta u=0$ mit vier reellen Variablen. Comment. Math. Helv. 1934, 7 (1), 307-330. doi:10.1007/BF01292723

[5] Gentili G., Struppa D.C. A new approach to Cullen-regular functions of a quaternionic variable. C. R. Math. Acad. Sci. Paris 2006, 342 (10), 741-744. doi:10.1016/j.crma.2006.03.015

[6] Gentili G., Struppa D.C. A new theory of regular functions of a quaternionic variable. Adv. Math. 2007,216 (1), 279-301. doi:10.1016/j.aim.2007.05.010

[7] Kim J.E. A polar representation of a regularity of a dual quaternionic function in Clifford analysis. Bull. Korean Math. Soc. 2017, 54 (2), 583-592. doi:10.4134/BKMS.b160200

[8] Kim J.E. Extensions for a regular function with values in dual quaternions. Acta Math. Acad. Paedagog. Nyházi. (N.S.) 2017, 33 (1), 31-38.

[9] Laville G., Ramadanoff I. Holomorphic cliffordian functions. Adv. Appl. Clifford Algebr. 1998, 8 (2), $323-340$. doi:10.1007/BF03043103

[10] Messelmi F. Analysis of dual functions. Annual Rev. Chaos Theory, Bifurcations, Dynamical Syst. 2013, 4, 37-54. doi:10.13140/2.1.1006.4006

[11] Sudbery A. Quaternionic analysis. Math. Proc. Cambridge Philos. Soc. 1979, 85 (2), 199-225. doi:10.1017/S0305004100055638

[12] Wang W. On non-homogeneous Cauchy-Fueter equations and Hartogs' phenomenon in several quaternionic variables. J. Geom. Phys. 2008, 58 (9), 1203-1210. doi:10.1016/j.geomphys.2008.04.004

Received 18.10.2020

Revised 30.12.2020

Кім Аж.І. Застосування регулярності зрізів до функцій дуальної кватерніонної змінної // Карпатські матем. публ. - 2021. - Т.13, №2. — С. 298-304.

У цій статті ми презентуємо алгебраїчні властивості дуальних кватерніонів і визначаємо регулярність зрізів функції дуальної кватерніонної змінної. Оскільки добуток дуальних кватерніонів $є$ некомутативним, то регулярність зрізів виводиться двома способами. Після цього ми розглядаємо рівняння Коші-Рімана та степеневі ряди, що відповідають дуальним кватерніонам.

Ключові слова і фрази: дуальне число, кватерніон, рівняння Коші-Рімана, регулярна шодо зрізів функція, аналіз Кліффрорда. 